\title{
An epigenetic predictor of death captures multi-modal measures of brain health
}

\author{
Robert F. Hillary ${ }^{1}$ - Anna J. Stevenson $\mathbb{D}^{1} \cdot$ Simon R. Cox $\mathbb{D}^{2,3} \cdot$ Daniel L. McCartney ${ }^{1} \cdot$ Sarah E. Harris $\mathbb{D}^{2,3} \cdot$ \\ Anne Seeboth $^{1}$ - Jon Higham ${ }^{4}$. Duncan Sproul ${ }^{4,5} \cdot$ Adele M. Taylor $^{2,3} \cdot$ Paul Redmond $^{2,3} \cdot$ Janie Corley ${ }^{2,3}$. \\ Alison Pattie ${ }^{2,3} \cdot$ Maria del. C. Valdés Hernández ${ }^{2,6} \cdot$ Susana Muñoz-Maniega ${ }^{2,6} \cdot$ Mark E. Bastin $^{2,6}$. \\ Joanna M. Wardlaw $\mathbb{D}^{2,6,7}$. Steve Horvath $\mathbb{1}^{8,9} \cdot$ Craig W. Ritchie $^{10} \cdot$ Tara L. Spires-Jones $^{7,11}$. \\ Andrew M. McIntosh $\mathbb{1}^{2,12} \cdot$ Kathryn L. Evans ${ }^{1,2} \cdot \operatorname{lan}$ J. Deary ${ }^{2,3} \cdot$ Riccardo E. Marioni ${ }^{1,2}$
}

Received: 30 July 2019 / Revised: 14 November 2019 / Accepted: 20 November 2019 / Published online: 3 December 2019

(c) The Author(s) 2019. This article is published with open access

\begin{abstract}
Individuals of the same chronological age exhibit disparate rates of biological ageing. Consequently, a number of methodologies have been proposed to determine biological age and primarily exploit variation at the level of DNA methylation (DNAm). A novel epigenetic clock, termed 'DNAm GrimAge' has outperformed its predecessors in predicting the risk of mortality as well as many age-related morbidities. However, the association between DNAm GrimAge and cognitive or neuroimaging phenotypes remains unknown. We explore these associations in the Lothian Birth Cohort 1936 ( $n=709$, mean age 73 years). Higher DNAm GrimAge was strongly associated with all-cause mortality over the eighth decade (Hazard Ratio per standard deviation increase in GrimAge: $1.81, P<2.0 \times 10^{-16}$ ). Higher DNAm GrimAge was associated with lower age 11 IQ $(\beta=-0.11)$, lower age 73 general cognitive ability $(\beta=-0.18)$, decreased brain volume $(\beta=-0.25)$ and increased brain white matter hyperintensities $(\beta=0.17)$. There was tentative evidence for a longitudinal association between DNAm GrimAge and cognitive decline from age 70 to 79 . Sixty-nine of 137 health- and brain-related phenotypes tested were significantly associated with GrimAge. Adjusting all models for childhood intelligence attenuated to non-significance a small number of associations (12/69 associations; 6 of which were cognitive traits), but not the association with general cognitive ability (33.9\% attenuation). Higher DNAm GrimAge associates with lower cognitive ability and brain vascular lesions in older age, independently of early-life cognitive ability. This epigenetic predictor of mortality associates with different measures of brain health and may aid in the prediction of age-related cognitive decline.
\end{abstract}

\section{Introduction}

The rapid ageing of the global population has resulted in an increase in the personal and societal burden of age-associated disease and disability [1]. Consequently, there is an urgent need to identify those individuals at high risk of age-related

These authors contributed equally: Robert F. Hillary, Anna J.

Stevenson

Supplementary information The online version of this article (https:// doi.org/10.1038/s41380-019-0616-9) contains supplementary material, which is available to authorized users.

Riccardo E. Marioni

riccardo.marioni@ed.ac.uk

Extended author information available on the last page of the article. morbidities and mortality. Recently, a number of methods for determining biological age have been developed which leverage inter-individual variation in physiological and molecular characteristics [2-6]. Primarily, these measures of biological age have focussed on variation at the level of DNA methylation (DNAm). DNAm is a commonly-studied epigenetic mechanism typically characterised by the addition of a methyl group to a cytosine-phosphate-guanine $(\mathrm{CpG})$ nucleotide base pairing, thereby permitting regulation of gene activity [7]. Crucially, these biological age predictors, also referred to as 'epigenetic clocks', correlate strongly with chronological age; furthermore, for a given chronological age, an advanced epigenetic age is associated with increased mortality risk and many age-related morbidities [8-12].

A novel epigenetic clock, termed 'DNAm GrimAge' has been developed to predict mortality [13]. To derive DNAm GrimAge, an elastic net Cox regression model was used to 
regress time-to-death due to all-cause mortality on chronological age, sex and DNAm-based surrogates for smoking pack years and 12 plasma proteins. The model selected chronological age, sex and methylation-based surrogates for smoking pack years and for $7 / 12$ plasma proteins. The linear combination of these variables allows for an estimation of DNAm GrimAge. As with other epigenetic clocks, if an individual's DNAm GrimAge is higher than their chronological age, then this provides a measure of accelerated biological ageing. Lu et al. [13] comprehensively demonstrated that an accelerated DNAm GrimAge (also known as AgeAccelGrim) is associated with a number of peripheral, lifestyle and cardiometabolic traits and outperforms predecessor clocks in predicting death. However, the relationship between an accelerated GrimAge and cognitive as well as neuroimaging phenotypes remains unexplored. As brain structure and cognitive function show mean declines with age, and associate with disability and disease burden, the discovery of molecular correlates of neurological and neurostructural aberrations may be of particular benefit in gerontology $[14,15]$. In this study we test the hypothesis that, in a large narrow age-range population cohort of older adults (Lothian Birth Cohort 1936 (LBC1936)), an accelerated DNAm GrimAge is crosssectionally associated with poorer cognitive performance, structural neuroimaging measures and neurology-related proteins.

In addition, higher childhood intelligence (as defined by age 11 IQ) is associated with a lower risk of mortality across the life course [16-18]. Furthermore, childhood intelligence associates with a healthier lifestyle and less morbidity in middle age, as well as a lower allostatic load in older age [19-21]. Intelligence in early life is related to variability in cortical thickness, white matter macro- and micro-structure, as well as cognitive ability, fewer vascular lesions and lower risk of stroke in later life [22-27]. Notably, adjustment for age 11 IQ was recently shown to attenuate associations between another epigenetic clock measure, DNAm PhenoAge, and a wide range of phenotypes including cognitive traits in LBC1936 [28]. Therefore, we also test the hypothesis that controlling for childhood intelligence attenuates associations between DNAm GrimAge and mortality, cognitive and neuroimaging measures, as well as neurology-related proteins in older age.

\section{Materials and methods}

\section{The Lothian Birth Cohort 1936}

The LBC1936 comprises Scottish individuals born in 1936, most of whom took part in the Scottish Mental Survey 1947 at age 11. Participants who were living within Edinburgh and the Lothians were re-contacted $\sim 60$ years later. Of these participants, 1091 consented and joined the LBC1936. Upon recruitment, participants were $\sim 70$ years of age (mean age: $69.6 \pm 0.8$ years) and subsequently attended four additional waves of clinical examinations about every 3 years. Detailed genetic, epigenetic, physical, psychosocial, cognitive, neuroimaging, health and lifestyle data are available for members of the LBC1936. Recruitment and testing of the LBC1936 have been described previously $[29,30]$.

\section{Methylation preparation in the Lothian Birth Cohort 1936}

DNA from whole blood was assessed using the Illumina $450 \mathrm{~K}$ methylation array at the Edinburgh Clinical Research Facility. Details of quality control procedures have been described elsewhere (see Supplementary Methods) [31, 32].

\section{Derivation of DNAm GrimAge}

DNAm GrimAge was calculated using the online age calculator (https://dnamage.genetics.ucla.edu/) developed by Horvath [33]. LBC1936 methylation data were used as input for the algorithm and data underwent a further round of normalisation by the age calculator. The DNAm GrimAge biomarker was calculated using a method developed by Lu et al. [13] and is based on a linear combination of age, sex, DNAm-based surrogates for smoking, and seven proteins (adrenomedulin (DNAm ADM), beta-2microglobulin (DNAm B2M), cystatin C (DNAm cystatin C), growth differentiation factor 15 (DNAM GDF15), leptin (DNAm leptin), plasminogen activation inhibitor 1 (DNAm PAI1), and tissue inhibitor metalloproteinaise (DNAm TIMP1)). Supplementary Fig. 1 shows the correlation between all methylation-based surrogates. All predictors, with the exception of DNAm Leptin $\left(r^{2}=-0.29\right)$, were positively correlated with DNAm GrimAge (absolute range $=[0.24: 0.82]$, median $=0.25$ and mean of correlation coefficients $=0.25$ ). The difference between DNAm GrimAge and chronological age (an accelerated DNAm GrimAge) provides a measure of biological ageing. In a previous study, for a given chronological age, individuals with higher DNAm GrimAge had a higher risk for mortality than individuals of the same chronological age with a lower DNAm GrimAge [13].

\section{Phenotypic data}

Our phenotypic analyses were divided into four sections. Firstly, we examined the association between age-adjusted DNAm GrimAge and mortality in the LBC1936 over 9 years of follow-up. For our survival models (and later 
longitudinal cognitive analyses), we aimed to determine whether DNAm GrimAge at Wave 1 of the LBC1936 study ( $n=906$; age: 70 years) could predict mortality (or cognitive decline) over all four waves of available data (to age 79 years). For all other phenotypic analyses, we examined cross-sectional associations with age-adjusted DNAm GrimAge at Wave 2 (age: 73 years). This is because complete proteomic, brain imaging, DNAm and phenotypic data were available at this time point only ( $n=709$ individuals). For cross-sectional analyses, we did not wish to determine whether Wave 1 (age: 70 years) epigenetic data associated with Wave 2 (age: 73 years) phenotypic data in order to limit the potential issue of retrocausality. In this first section, we also investigated the cross-sectional association of an accelerated DNAm GrimAge with a number of physical (body mass index, height, grip strength, lung function and weight) and blood traits (albumin, C-reactive protein, cholesterol, creatinine, ferritin, interleukin-6 and iron; at Wave 2; age 73 years) that have been related to mortality and frailty in older age [34-42].

Secondly, we tested the association between an accelerated DNAm GrimAge and cognitive traits $(n=18$ phenotypes). Cognitive tests taken at Wave 2 (age: 73 years) included six Wechsler Adult Intelligence Scale-III UK (WAIS-III) non-verbal subtests (matrix reasoning, letter number sequencing, block design, symbol search, digit symbol, and digit span backward). Principal component analysis (PCA) was performed using these cognitive tests and scores on the first un-rotated principal component (general cognitive ability, $g$ ) were extracted which explained $51 \%$ of variance. Individual test loadings ranged from 0.65 to 0.75 . Wechsler Memory Scale-III items as well as measures of crystallised intelligence and reaction time were also examined in relation to DNAm GrimAge. In addition, we examined whether an accelerated DNAm GrimAge associated with APOE \&4 carrier status. Similar to our survival analyses, we used Wave 1 epigenetic data to determine whether DNAm GrimAge (at age 70 years) could predict decline in general cognitive ability across all four waves of the LBC1936 study. For this analysis, we used the lmerTest package in R to fit mixed-effects models to regress general cognitive ability onto sex and an interaction term between DNAm GrimAge at Wave 1 and chronological age, all as fixed effects [43]. In addition, participant ID was fitted as a random effect on the intercept.

Thirdly, we tested the association between an accelerated DNAm GrimAge and neuroimaging phenotypes at Wave 2 (age: 73 years, see Supplementary Methods). The brain MRI acquisition and processing pipeline has been made available in an open access protocol paper [44]. Total brain, normal-appearing white matter, grey matter and white matter hyperintensity volumes were segmented using a semi-automated multi-spectral technique [45]. These volumes were then expressed as a proportion of intracranial volume (ICV), which controls for the confounding effect of head size. The resultant ratios were tested for associations with age-adjusted DNAm GrimAge. Diffusion-tensor imaging-derived measures of fractional anisotropy (FA) and mean diffusivity (MD) were obtained for participants at Wave 2 (age: 73 years). Prior to conducting region-specific analyses, general factors of FA ( $\mathrm{gFA}$ ) and MD (gMD) were derived by entering the left and right $F A$ and MD values of each tract separately into a PCA. Scores from the first un-rotated principal component were extracted and labelled as gFA (variance explained: $52 \%$, loadings: $0.46-0.95$ ) or gMD (variance explained: $48 \%$, loadings: $0.47-0.88$ ), respectively. These general factors reflect common microstructural properties across main white matter pathways and capture the common variance in white matter integrity [46].

Fourthly, we tested the association between an accelerated DNAm GrimAge and the levels of 92 neurological protein biomarkers $\left(\right.$ Olink $^{\circledR}$ neurology panel). The neurology panel represents proteins with established links to neuropathology as well as exploratory proteins with roles in processes including cellular communication and immunology. Plasma was extracted from 816 blood samples collected in citrate tubes at mean age $72.5 \pm 0.7$ years (Wave 2; Supplementary Methods). Protein levels were transformed by rank-based inverse normalisation. Normalised protein levels were regressed onto age-adjusted DNAm GrimAge.

Descriptive statistics for phenotypes are presented in Supplementary File 1. Data collection protocols have been described fully previously and are described in Supplementary Note 1 [47].

\section{Statistical analyses}

DNAm GrimAge was regressed onto chronological age for all LBC1936 participants. These residuals were defined as an accelerated DNAm GrimAge (also known as AgeAccelGrim). Linear regression models were used to investigate relationships between continuous variables and an accelerated DNAm GrimAge, as well as age-adjusted methylationbased surrogates for smoking pack years and the plasma proteins that feed into DNAm GrimAge. Logistic regression was used to test the association between methylation-based predictors and $A P O E$ \&4 carrier status. An accelerated DNAm GrimAge, age-adjusted DNAm Pack Years or ageadjusted DNAm plasma protein levels were the independent variable of interest in each regression model and all variables were scaled to have a mean of zero and unit variance. Height and smoking status were included as covariates in the models for lung function (forced expiratory volume FEV1; forced vital capacity: FVC; forced expiratory ratio: FER; and peak expiratory flow: PEF). All models were adjusted for chronological age and sex. Mixed-effects 
models were used to examine the longitudinal association between an accelerated DNAm GrimAge and general cognitive ability. To investigate possible statistical confounding by childhood cognitive ability, all models were repeated with adjustment for age 11 IQ scores. To correct for multiple testing, and given that the methylation-based predictors exhibited a high degree of inter-correlation, we applied the false discovery rate (FDR; [48]) method to phenotypic association analyses $(n=137$ phenotypes), separately for each predictor. Associations between ageadjusted DNAm GrimAge and regional cortical volume were conducted using the SurfStat toolbox (http://www.ma th.mcgill.ca/keith/surfstat) for Matrix Laboratory R2018a (The MathWorks Inc, Natick, MA), using the same covariates as above and FDR correction for multiple testing.

\section{Results}

\section{Cohort characteristics}

Details of LBC1936 participant characteristics at Waves 1 and 2 are presented in Supplementary File 1. Briefly, 47.6\% of participants in this study were female. At Wave 1 (relating to the mortality and longitudinal analyses), mean chronological age for both males and females was 69.6 years (SD 0.8), whereas the mean DNAm GrimAge was 67.4 years (SD 5.2). At Wave 2 (relating to cross-sectional analyses), mean chronological age for both males and females was 72.5 years (SD 0.7), whereas the mean DNAm GrimAge was 70.0 years (SD 4.9). The lower mean measure of epigenetic age when compared to chronological age may reflect overall good health of the cohort. However, the variance associated with DNAm GrimAge is much higher than that of chronological age. When calculated across all four available waves of the LBC1936 study, DNAm GrimAge exhibits an intra-class correlation coefficient of 0.85 . Mean age 11 IQ scores were 100.69 (SD: 15.37). Notably, lower IQ scores at age $11(\beta=-0.11, P=0.02)$ were associated with an accelerated DNAm GrimAge. Associations between age 11 IQ and tested phenotypes are presented in Supplementary File 2.

\section{DNAm GrimAge predicts mortality and associates with frailty factors in the LBC1936}

Mortality in LBC1936 participants was assessed in relation to an accelerated DNAm GrimAge as well as age-adjusted DNAm-based surrogate markers for plasma protein levels and smoking pack years. DNAm GrimAge was derived for 906 participants with methylation data (at Wave 1: age 70 years). There were 226 deaths $(24.9 \%)$ over 9 years of follow-up.
A higher DNAm GrimAge was significantly associated with risk of all-cause mortality (Hazard Ratio $(\mathrm{HR})=1.81$ per SD increase in DNAm GrimAge, 95\% confidence interval $\left.(\mathrm{CI})=[1.58,2.07], P<2.0 \times 10^{-16}\right)$. Furthermore, higher levels of age-adjusted DNAm Pack Years were associated with all-cause mortality in the LBC1936 (HR = 1.64 per SD, $95 \%$ CI $[1.46,1.86], P=2.0 \times 10^{-16}$ ). In relation to methylation-based surrogates for plasma protein levels, six of the seven DNAm protein surrogates (DNAm ADM, B2M, Cystatin C, GDF15, PAI1 and TIMP1) were significantly associated with all-cause mortality (see Supplementary File 3; Fig. 1a). Following adjustment for age $11 \mathrm{IQ}$, there was very little change in the HRs and all of the predictors remained significant. Indeed, HRs from all survival models ranged from an attenuation of $2.4 \%$ to an increase of $1.8 \%$ following adjustment for childhood intelligence.

A Kaplan-Meier survival plot for an accelerated DNAm GrimAge, split into the highest and the lowest quartiles, is presented in Fig. 1b illustrating the higher mortality risk for those with a higher DNAm GrimAge. Kaplan-Meier survival plots for methylation-based surrogates for smoking pack years and plasma protein levels are presented in Supplementary Fig. 2.

For the remainder of the results, only those associations with an FDR-corrected significant $\mathrm{P}$ value $(<0.05)$ are presented herein and in Fig. 2. Full results are presented in Supplementary File 4. In relation to major mortality- and frailty-associated physical traits in the LBC1936, an accelerated DNAm GrimAge was associated with increased levels of interleukin-6 $\left(\beta=0.37, P=2.3 \times 10^{-18}\right)$, C-reactive protein $\left(\beta=0.25, P=2.8 \times 10^{-8}\right)$, creatinine $(\beta=0.16, P=$ $\left.1.1 \times 10^{-4}\right)$, an increased body mass index $(\beta=0.16, P=$ $\left.2.9 \times 10^{-4}\right)$, triglyceride concentration $(\beta=0.13, P=5.0 \times$ $\left.10^{-3}\right)$ and body weight $(\beta=0.09, P=0.04)$ (Fig. 2). The relationship between accelerated DNAm GrimAge and triglycerides was no longer significant after controlling for childhood cognitive ability with the effect size decreasing from 0.13 to 0.09 (32.5\% attenuation) (Supplementary File 4).

An accelerated DNAm GrimAge was negatively associated with all four measures of lung function $(\beta=[-0.16$ to -0.27$], P=\left[9.4 \times 10^{-7}\right.$ to $\left.\left.1.7 \times 10^{-16}\right]\right)$, iron levels $\left(\beta=-0.24, \quad P=7.2 \times 10^{-7}\right), \quad$ low-density lipoprotein cholesterol levels $\left(\beta=-0.17, P=1.1 \times 10^{-4}\right)$, total cholesterol levels $\left(\beta=-0.13, P=1.1 \times 10^{-4}\right)$ and height $(\beta=-0.08, P=0.01)$ (Fig. 2). Only the relationship between accelerated DNAm GrimAge and height was non-significant after controlling for childhood intelligence, with the effect size attenuating from -0.08 to -0.06 (\% attenuation: $24.5 \%$ ) (Supplementary File 4). On average, associations were attenuated by $2.5 \%$ after controlling for age 11 IQ [ranged from: $19.1 \%$ increase (total 
A

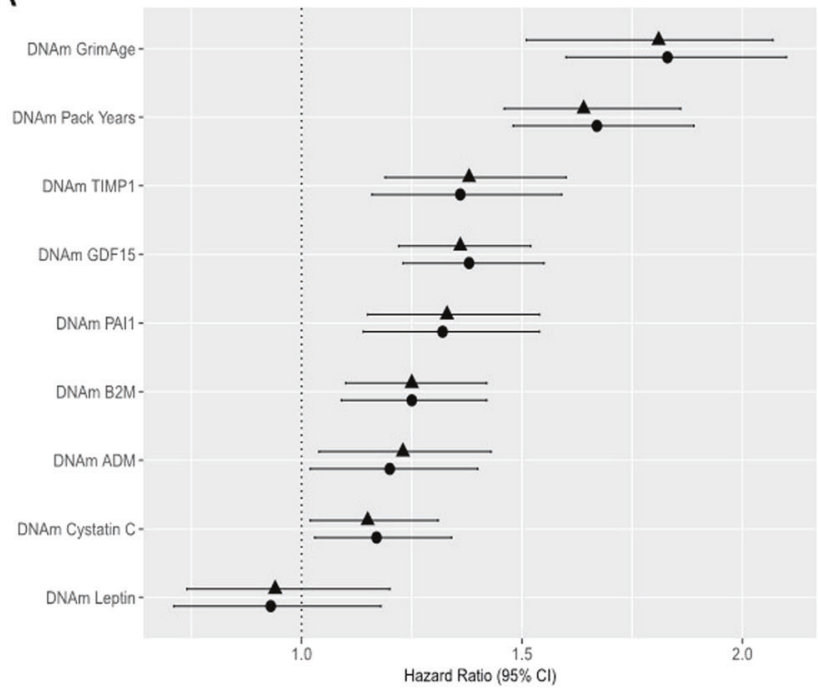

Fig. 1 DNAm GrimAge and its component surrogate markers predict mortality in the LBC1936. a Forest plot showing hazard ratios and 95\% confidence intervals (horizontal lines) from Cox proportional hazard models for DNAm GrimAge and its constituent DNAm surrogate markers in the LBC1936 $(n=906$, no. of deaths $=226$
B

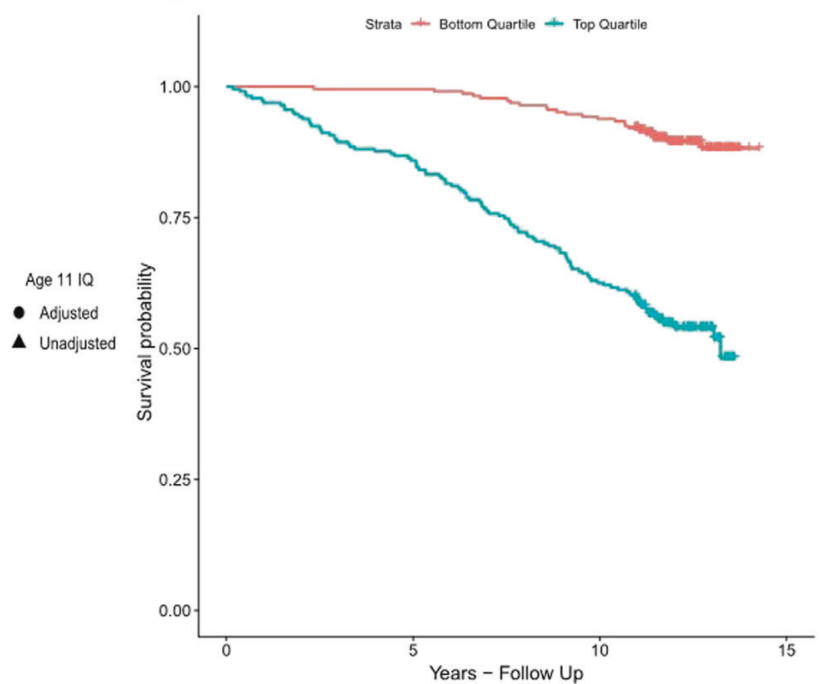

following nine years of follow-up). All associations with the exceptions of DNAm Leptin were significant. b Kaplan-Meier survival curve exhibiting the survival probabilities for the top (highest DNAm GrimAge) and bottom quartiles (lowest DNAm GrimAge) for DNAm GrimAge in the LBC1936 following 9 years of follow-up
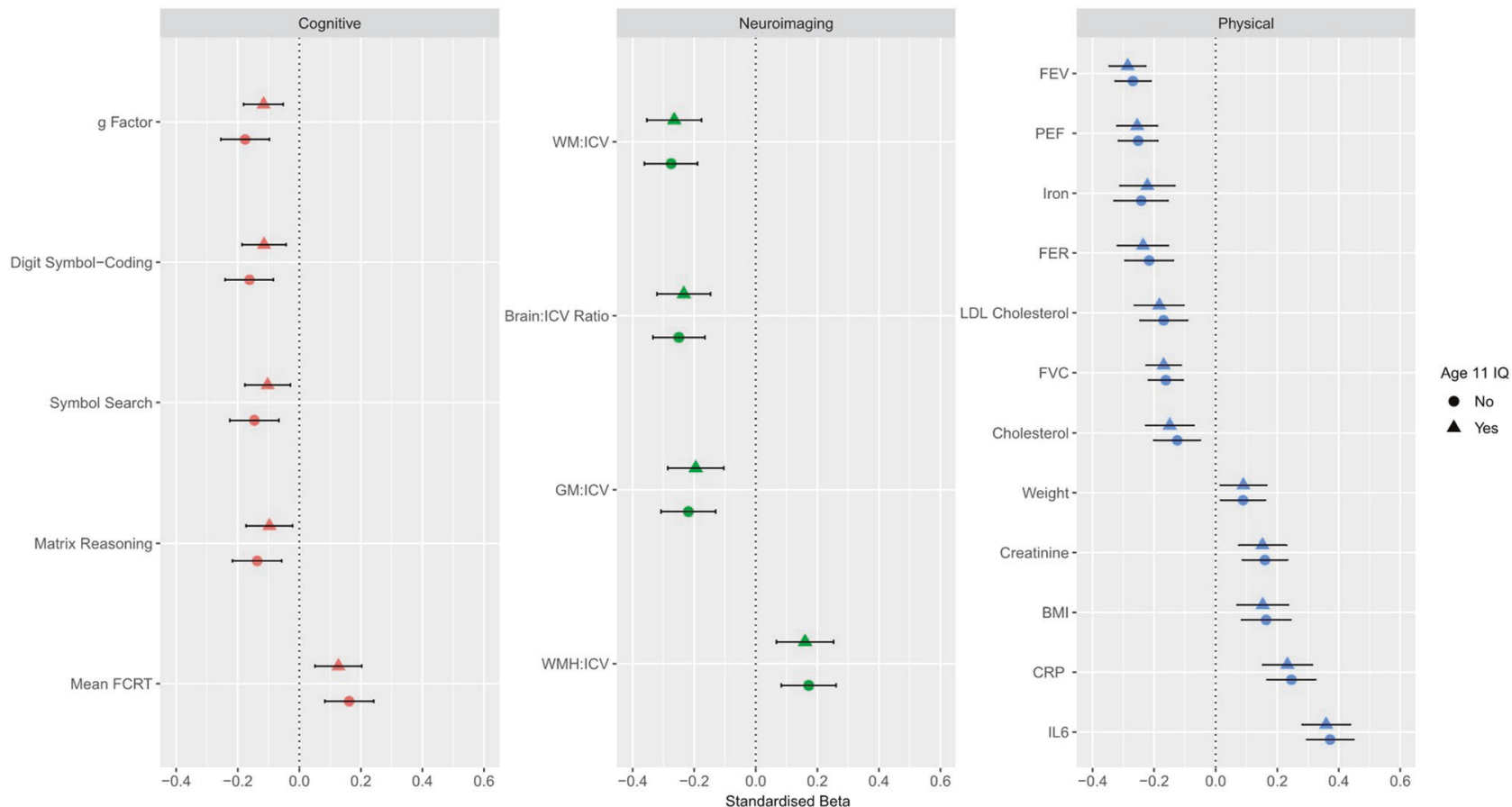

Fig. 2 Cross-sectional association between age-adjusted DNAm GrimAge and cognitive, neuroimaging and physical traits in the LBC1936. Cognitive: An accelerated DNAm GrimAge was negatively associated with the general factor of cognitive ability, digit symbol coding, symbol search and matrix reasoning tasks. DNAm GrimAge was also associated with an increased mean four choice reaction time. Neuroimaging: Age-adjusted DNAm GrimAge was negatively associated with the ratios of white matter volume, brain volume and grey matter volume to intracranial volume, and positively associated with the ratio of volume of white matter hyperintensities to intracranial volume. Physical: An accelerated DNAm GrimAge was negatively associated with four measures of lung function: forced expiratory

volume in $1 \mathrm{~s}$, forced vital capacity, forced expiratory ratio and peak expiratory flow, as well as levels of iron, low-density lipoprotein cholesterol and total cholesterol. Age-adjusted DNAm GrimAge was positively associated with weight, levels of creatinine, body mass index as well as levels of C-reactive protein and interleukin-6. Horizontal lines indiciate 95\% confidence intervals. BMI body mass index, CRP C-reactive protein, FCRT four choice reaction time, FER forced expiratory ratio, FEV forced expiratory volume, FVC forced vital capacity, GM grey matter, ICV intracranial volume, IL6 interleukin-6, LDL low-density lipoprotein, PEF peak expiratory flow, WM white matter, WHM white matter hyperintensities 
cholesterol) to $32.5 \%$ attenuation (triglycerides)]. All associations between blood and physical traits and an accelerated DNAm GrimAge in this study are presented in Supplementary Fig. 3. Relationships between all phenotypes tested in this study and age-adjusted DNAm Pack Years as well as age-adjusted plasma protein levels are presented in Supplementary File 5. Significant relationships are further detailed in Supplementary Note 2.

\section{DNAm GrimAge associates with lower cognitive ability in the LBC1936}

An accelerated DNAm GrimAge was significantly associated with lower measures of general cognitive ability (g: $\beta=-0.18, P=8.0 \times 10^{-6} ; n=709$ ). Furthermore, an accelerated DNAm GrimAge was negatively associated with all six component tests for fluid intelligence from which $g$ was derived (see Section "Phenotypic data"; $\beta=[-0.11$ to $-0.16], P=\left[0.02\right.$ to $\left.\left.2.4 \times 10^{-4}\right]\right)$. In addition, an accelerated DNAm GrimAge was associated with an increased four choice reaction time mean $\left(\beta=0.16, P=2.9 \times 10^{-4}\right)$. Lower IQ scores at age 70 (which correlated 0.70 with age 11 IQ scores) were associated with age-adjusted DNAm GrimAge $(\beta=-0.11, P=0.02)$. An accelerated DNAm GrimAge was also negatively associated with the following measures of crystallised intelligence: the Wechsler Test of Adult Reading $\left(\beta=-0.13, P=4.0 \times 10^{-3}\right)$ and the National Adult Reading Test $(\beta=-0.10, P=0.03)$.

Following adjustment for age $11 \mathrm{IQ}$, an accelerated DNAm GrimAge remained significantly associated with general cognitive ability $\left(g: \beta=-0.12, P=2.0 \times 10^{-3}\right.$; $33.9 \%$ attenuation). Three out of the six tests which constitute the general intelligence factor remained significant after adjustment for age 11 IQ (digit-symbol coding, symbol search, and matrix reasoning). Furthermore, the association between an accelerated DNAm GrimAge and an increased mean four choice reaction time remained significant following adjustment for age 11 IQ (Fig. 2). On average, associations between cognitive tasks and an accelerated DNAm GrimAge were attenuated by $41.1 \%$ following controlling for age 11 IQ (ranging from $21.7 \%$ attenuation [four choice reaction time] to $77.4 \%$ attenuation [National Adult Reading Test]). All associations between cognitive traits and an accelerated DNAm GrimAge in this study are presented in Supplementary Fig. 4. Finally, an accelerated DNAm GrimAge was not associated with APOE $\varepsilon 4$ carrier statusthe strongest genetic risk factor for Alzheimer's disease (odds ratio $=0.96,95 \% \mathrm{CI}=[0.93,1.00], P=0.06$ ).

Accelerated DNAm GrimAge showed a borderline significant association with faster cognitive decline (interaction term between an accelerated DNAm GrimAge at Wave 1 and age: $\beta=-0.018, P=0.05 ; n=906)$. This association was attenuated following adjustment for age $11 \mathrm{IQ}$
( $\beta=-0.015, P=0.11, \%$ attenuation: $16.7 \%)$. Secondly, restricting the set of individuals to just those incorporated into our cross-sectional design $(n=709)$, accelerated DNAm GrimAge at Wave 1 was significantly associated with decline in general cognitive ability across the eighth decade $(\beta=-0.020, P=0.03 ; n=709)$. After adjusting for childhood cognitive ability, this association was attenuated to non-significance $(\beta=-0.017, P=0.07, \%$ attenuation: $15 \%)$.

\section{DNAm GrimAge is associated with gross neurostructural differences in the LBC1936}

An accelerated DNAm GrimAge was associated with lower white matter volume $\left(\beta=-0.28, P=1.7 \times 10^{-8}\right)$, total brain volume $\left(\beta=-0.25, P=1.4 \times 10^{-7}\right)$ and grey matter volume $\left(\beta=-0.22, \quad P=1.3 \times 10^{-5}\right)$. Furthermore, an accelerated DNAm GrimAge was associated with an increased volume of white matter hyperintensities $(\beta=$ $0.17, P=1.0 \times 10^{-3}$ ) (Fig. 2). All associations remained significant following adjustment for age 11 IQ (Supplementary File 4). On average, these associations were attenuated by $6.98 \%$ after adjusting for age 11 IQ. All associations between neuroimaging traits and an accelerated DNAm GrimAge in this study are presented in Supplementary Fig. 5.

An accelerated DNAm GrimAge was not significantly associated with general factors of white matter microstructural metrics i.e. fractional anisotropy $(\beta=-0.009$, $P=0.89$ ) or mean diffusivity $(\beta=-0.001, P=0.98)$, hence additional regional analyses were not performed. However, given that DNAm GrimAge was associated with grey matter volume, we further tested whether there was regional cortical heterogeneity in relation to the DNAm GrimAge-grey matter association. The negative association between accelerated DNAm GrimAge and cortical volume showed a degree of regional heterogeneity across the cortical surface (Fig. 3). The strongest magnitudes were evident in lateral and medial frontal and temporal regions, extending into motor and somatosensory cortex as well as into the posterior cingulate and precuneal areas. In contrast, associations in occipital and inferior lateral and medial frontal regions were non-significant. When the associations were additionally corrected for age $11 \mathrm{IQ}$, the magnitude of the effect sizes at the FDR-significant loci were weakly attenuated (mean $t$-value attenuation $=3.36 \%$; Supplementary Fig. 6).

\section{Association of DNAm GrimAge with neurological protein biomarkers}

Forty of the 92 neurology-related Olink $^{\circledR}$ proteins were significantly associated with an accelerated DNAm GrimAge at FDR-corrected $P<0.05 \quad(n=709)$. These 

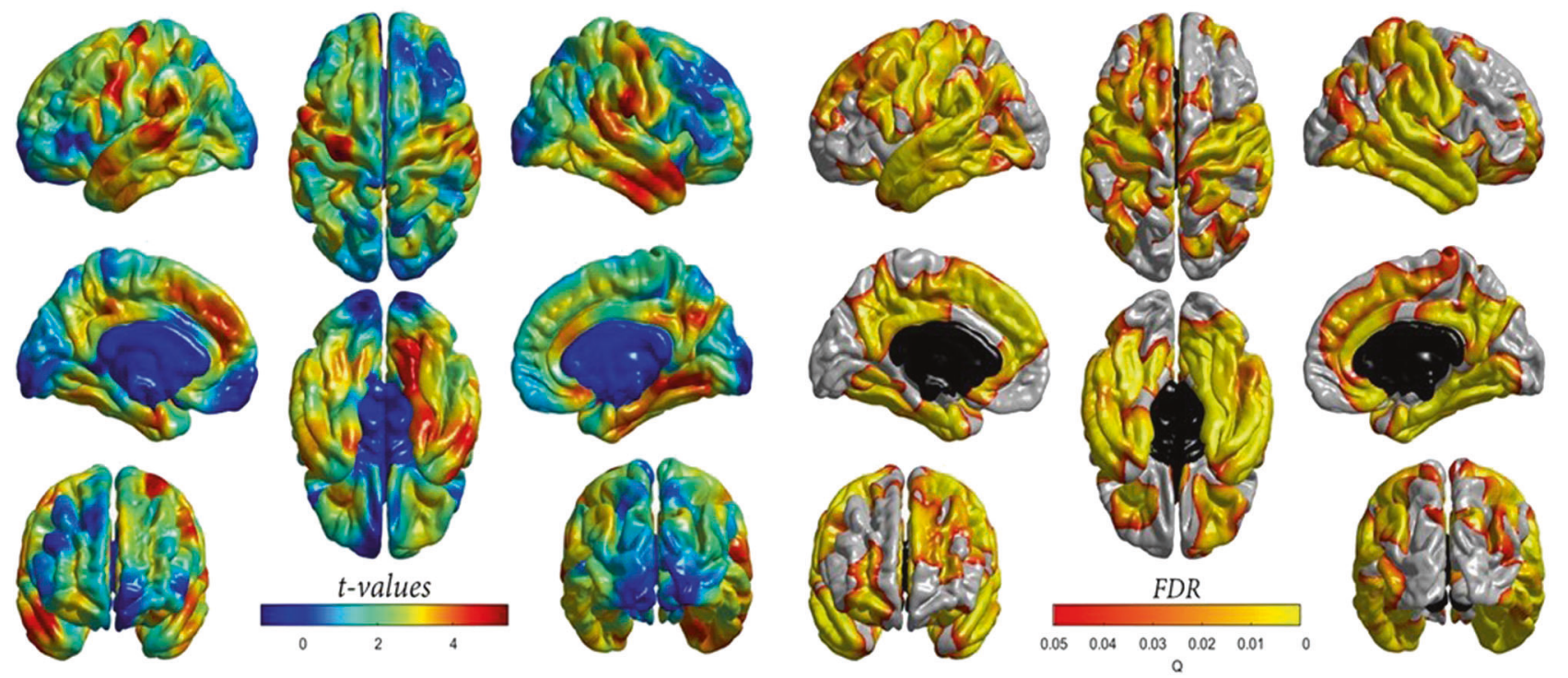

Fig. 3 Cross-sectional association between age-adjusted DNAm GrimAge and regional cortical volume in the LBC1936. Left panel: $t$ values indicate the magnitude of the negative association (values have been flipped for visualisation purposes). An accelerated DNAm

GrimAge was negatively associated with cortical volume. Right panel: Corresponding FDR-corrected $P$ values indicate the spatial distribution of significant associations. FDR false discovery rate

proteins explained between $0.73 \%(\beta=-0.09$, NC-Dase $)$ to $7.19 \%(\beta=0.30$, SKR3) of inter-individual variation in an accelerated DNAm GrimAge (in a model which was not adjusted for age and sex; Supplementary File 6). Following adjustment for age 11 IQ, 36/40 associations (90\%) remained significant. After adjusting for age $11 \mathrm{IQ}$, associations were, on average, attenuated by $3.03 \%$.

\section{Correlation between DNAm GrimAge and DNAm Pack Years}

We observed that DNAm GrimAge and DNAm Pack Years were highly correlated (correlation coefficient: 0.82 ) and were cross-sectionally associated with many of the same variables in our phenotypic analyses (Supplementary File 7). Therefore, we carried out a follow-up analysis to determine the difference in magnitude between the effect sizes for DNAm GrimAge or DNAm Pack Years in relation to phenotypes associated with both predictors. Prior to adjusting for age $11 \mathrm{IQ}$, the effect sizes had a correlation coefficient of 0.88 . However, they were, on average, $16.5 \%$ greater for DNAm GrimAge when compared to DNAm Pack Years. Following adjustment for age $11 \mathrm{IQ}$, the correlation coefficient was 0.84 , and the effect sizes were, on average, $23.1 \%$ greater for DNAm GrimAge upon comparison to DNAm Pack Years. A plot demonstrating the correlation between effect sizes for DNAm GrimAge and DNAm Pack Years from our crosssectional phenotypic analyses is presented in Supplementary Fig. 7.

\section{Sex-specific differences in associations with DNAm GrimAge}

As a sensitivity analysis, we accounted for an interaction between age-adjusted DNAm GrimAge and sex. Prior to adjusting for age $11 \mathrm{IQ}$, there was evidence for a sex-specific difference only in the relationships between an accelerated DNAm GrimAge and all four measures of lung function (FVC: $\quad \beta_{\text {GrimAge } \times \text { males }}=0.50, \quad P_{\text {GrimAge } \times \text { males }}=5.6 \times 10^{-39}$; FEV: $\quad \beta_{\text {GrimAge } \times \text { males }}=0.39, \quad \mathrm{P}_{\text {GrimAge } \times \text { males }}=1.3 \times 10^{-23}$; PEF: $\beta_{\text {GrimAge } \times \text { males }}=0.17, \mathrm{P}_{\text {GrimAge } \times \text { males }}=1.2 \times 10^{-4}$; FER: $\left.\beta_{\text {GrimAge } \times \text { males }}=-0.14, \mathrm{P}_{\text {GrimAge } \times \text { males }}=0.049\right)$ (Supplementary File 8 ). The same interaction model was also rerun accounting for age $11 \mathrm{IQ}$. Three of the lung function tests (all but FER), as well as reading ability and general cognitive ability, exhibited significant interactions between sex and DNAm GrimAge (Supplementary File 9).

\section{Adjustment for educational attainment}

In a further sensitivity analysis, we found that an accelerated DNAm GrimAge was significantly associated with years of education $\left(\beta=-0.12, P=1.7 \times 10^{-3}\right)$. Models adjusted for age 11 IQ were rerun with an additional adjustment for years of education. Of the 57 relationships which remained significant after adjusting for age 11 IQ, eight were attenuated to non-significance when adjusting for education. These included associations with symbol search and weight $(\beta=-0.10$ to $-0.08, \%$ attenuation $=22.3 \% ; \beta=0.09$ to $0.05, \%$ attenuation $=44.4 \%$, respectively) and with six proteins 
(THY 1, RGMA, CDH3, TNFRSF21, NEP and TMPRSS5, mean attenuation: 8.8\%) (Supplementary File 10).

\section{Discussion}

In this study, we found that a higher-than-expected DNAm GrimAge strongly predicted mortality and was associated with a number of mortality- and frailty-associated traits. This provides the first external replication of the association between DNAm GrimAge and survival. After controlling for childhood cognitive ability, we found that an accelerated DNAm GrimAge was cross-sectionally associated with lower general cognitive ability as well as slower reaction time speed and lower scores on processing speed and perceptual organisation tasks. There was tentative evidence to suggest that an accelerated DNAm GrimAge measured at age 70 may predict decline in general cognitive ability up to age 79. Furthermore, an accelerated DNAm GrimAge was associated with gross neuroanatomical differences and vascular lesions in older age. Finally, a number of neurology-related proteins were associated with an accelerated DNAm GrimAge.

DNAm GrimAge was developed using mortality as a reference and consequently supplants its predecessors in relation to mortality risk prediction. Indeed, in this study, we observed a hazard ratio of 1.81 per standard deviation increase in an accelerated DNAm GrimAge, which outperforms that of previous epigenetic clocks (Hannum Age HR: 1.22, Horvath Age HR: 1.19; DNAm PhenoAge HR: 1.17; all applied to LBC1936) [8, 28]. In relation to mortality- and frailty-associated traits, the strongest association was between DNAm GrimAge and interleukin-6. Furthermore, DNAm GrimAge was strongly associated with C-reactive protein (whose production is stimulated by interleukin-6). Together, this corroborates evidence for the "inflammaging" theory which postulates that chronic, low-grade inflammation significantly influences biological ageing and decline [49]. An accelerated DNAm GrimAge was also associated with lower low-density lipoprotein cholesterol and total cholesterol. In older age, lower levels of these blood-based factors are also associated with higher risk of mortality [50]. In addition, DNAm GrimAge was associated with a higher body mass index which does not agree with previous findings showing that an increased body mass index is protective against mortality risk [39]. However, this may be driven by a strong association between DNAm Leptin and body mass index. Indeed, leptin is an adipose tissue-derived hormone which acts an appetite suppressant, and is strongly correlated with body mass index and obesity [51, 52].

We observed a significant relationship between higher childhood intelligence (as well as age 70 IQ) and a lower
DNAm GrimAge in older age. After controlling for childhood cognitive ability, associations between DNAm GrimAge and tests of crystallised intelligence were attenuated to non-significance. This finding is not surprising given that crystallised intelligence remains stable throughout adulthood [53], and that the National Adult Reading Test strongly retrodicts childhood IQ in this sample [54]. However, relationships between DNAm GrimAge and general cognitive ability, as well as fluid intelligence measures, remained significant after adjusting for age 11 IQ. Nevertheless, these associations were attenuated by an average of $41.4 \%$ following adjustment for age 11 IQ. Therefore, blood-based methylation changes, as captured by DNAm GrimAge, helps to explain additional variance in late life cognitive ability and fluid intelligence.

An accelerated DNAm GrimAge was significantly associated with gross neurostructural differences, including reductions in total brain, grey matter and white matter volumes and increases in white matter hyperintensity volumes. There was also some heterogeneity in the associations with regional cortical volume, whereby effects were strongest in frontal and temporal regions. These regions also exhibit the largest annual decrease in middle and older age [55], and are most informative for predicting chronological age (albeit using cortical thickness rather than volume; [56]). White matter hyperintensities, which associate with DNAm GrimAge, have also been linked to cortical loss in temporal and lateral frontal regions [57]. This may indicate that altered methylation profiles could help explain mechanistic relationships between neurovascular lesions and cortical atrophy. However, adjustment for vascular risk factors such as hypercholesterolaemia, smoking and diabetes is merited in this context. Furthermore, white matter hyperintensities are also related to physical disability, processing speed and cognitive decline [58,59]. Additionally, the presence of white matter hyperintensities doubles the risk of dementia, and triples the risk of stroke, and is associated with clinical outcomes in stroke $[60,61]$. Therefore, DNAm GrimAge may capture vital aspects of age-related alterations in neurostructural integrity and gross brain pathology.

Here, DNAm GrimAge associated with poorer cognitive ability and neurostructural correlates of dementia. Dementia encompasses strong psychiatric components and overlaps with other psychiatric conditions [62]. In addition, there is a significant genetic or phenotypic overlap between cognitive ability and psychiatric conditions, such as schizophrenia and depression [63, 64]. Furthermore, DNAm GrimAge captured various deleterious aspects of brain health, including altered brain structure and neurological protein biomarkers, which relate to psychiatric disorders. Thus, this composite molecular predictor of mortality should be measured in other large-scale 
cohorts with incident and prevalent neurological and neuropsychiatric phenotype data to determine its utility in predicting clinically-defined disease.

We observed a very strong correlation between DNAm GrimAge and DNAm Pack Years. Indeed, the associations between smoking and mortality, cognitive decline and brain pathology are well-documented [65-67]. However, the larger effect sizes for DNAm GrimAge suggest that this composite biomarker is supplemented by the inclusion of methylation-based surrogates for plasma protein levels. We identified associations with a number of neurology-related proteins $(n=40$ before adjustment for age $11 \mathrm{IQ} ; n=36$ after adjustment for age 11 IQ) which may further inform the risk of mortality and age-related morbidities, particularly in relation to neurological disease. Future studies are necessary to define the biological relationships between such proteins and their relevance to age-related pathologies and cognitive decline.

The use of methylation-based proxies for smoking pack years and proteomic data is advantageous as methylationbased predictors are often more accurate than self-reported phenotypes, and the cost of complex proteomic platforms is negated [68]. One strength of this study is that rich data were available across the eighth decade of life, a period in which risk of cognitive decline and compromised brain integrity increases significantly. However, LBC1936 comprises relatively healthy older adults, complicating the generalisability of findings to at-risk clinical populations and broader age ranges.

In conclusion, we demonstrated that an epigenetic predictor of mortality associates with cognitive ability, cognitive decline and neuroimaging phenotypes in a cohort of healthy older ageing adults. These associations were largely independent of another well-known predictor of mortality, childhood intelligence. Indeed, methylation alterations in blood, as captured by DNAm GrimAge, could help provide early indications towards mortality prediction and decline in brain health.

\section{Code availability}

Code will be available from the authors on request.

Acknowledgements We thank the LBC1936 participants and study team. We thank the nursing staff at the Wellcome Trust Clinical Research Facility, and radiographers at the Brain Research Imaging Research Centre (www.bric.ed.ac.uk), Western General Edinburgh. The LBC1936 is funded by Age UK as The Disconnected Mind project, and by the Medical Research Council (G0701120, G1001245, MR/M013111/1, MR/R024065/1). SRC, MEB and IJD are also supported by a National Institutes of Health (NIH) research grant R01AG054628. RFH and AJS are supported by funding from the Wellcome Trust 4-year PhD in Translational Neuroscience-training the next generation of basic neuroscientists to embrace clinical research [108890/Z/15/Z]. AS is supported by a Medical Research
Council PhD Studentship in Precision Medicine with funding by the Medical Research Council Doctoral Training Programme and the University of Edinburgh College of Medicine and Veterinary Medicine. DLM and REM are supported by Alzheimer's Research UK major project grant ARUK-PG2017B-10. J.M.W is supported by the Fondation Leducq. SH is supported by 1U01AG060908 - 01 .

\section{Compliance with ethical standards}

Conflict of interest The authors declare that they have no conflict of interest.

Ethical approval Ethical permission for LBC1936 was obtained from the Multi-Centre Research Ethics Committee for Scotland (MREC/01/ 0/56), the Lothian Research Ethics Committee (Wave 1: LREC/2003/ 2/29) and the Scotland A Research Ethics Committee (Waves 2, 3 and 4: 07/MRE00/58).

Informed consent Written informed consent was obtained from all participants.

Publisher's note Springer Nature remains neutral with regard to jurisdictional claims in published maps and institutional affiliations.

Open Access This article is licensed under a Creative Commons Attribution 4.0 International License, which permits use, sharing, adaptation, distribution and reproduction in any medium or format, as long as you give appropriate credit to the original author(s) and the source, provide a link to the Creative Commons license, and indicate if changes were made. The images or other third party material in this article are included in the article's Creative Commons license, unless indicated otherwise in a credit line to the material. If material is not included in the article's Creative Commons license and your intended use is not permitted by statutory regulation or exceeds the permitted use, you will need to obtain permission directly from the copyright holder. To view a copy of this license, visit http://creativecommons. org/licenses/by/4.0/.

\section{References}

1. Vos T, Flaxman AD, Naghavi M, Lozano R, Michaud C, Ezzati M, et al. Years lived with disability (YLDs) for 1160 sequelae of 289 diseases and injuries 1990-2010: a systematic analysis for the Global Burden of Disease Study 2010. Lancet. 2012;380:2163-96.

2. Levine ME, Lu AT, Quach A, Chen BH, Assimes TL, Bandinelli $\mathrm{S}$, et al. An epigenetic biomarker of aging for lifespan and healthspan. Aging. 2018;10:573-591.

3. Horvath S. DNA methylation age of human tissues and cell types. Genome Biol. 2013;14:R115-R115.

4. Hannum G, Guinney J, Zhao L, Zhang L, Hughes G, Sadda S, et al. Genome-wide methylation profiles reveal quantitative views of human aging rates. Mol Cell. 2013;49:359-367.

5. Cole JH, Ritchie SJ, Bastin ME, Valdés Hernández MC, Muñoz Maniega S, Royle N, et al. Brain age predicts mortality. Mol Psychiatry. 2017;23:1385.

6. Vanhooren V, Dewaele S, Libert C, Engelborghs S, De Deyn PP, Toussaint $\mathrm{O}$, et al. Serum N-glycan profile shift during human ageing. Exp Gerontol. 2010;45:738-43.

7. Beck S, Rakyan VK. The methylome: approaches for global DNA methylation profiling. Trends Genet. 2008;24:231-7.

8. Marioni RE, Shah S, McRae AF, Chen BH, Colicino E, Harris SE, et al. DNA methylation age of blood predicts all-cause mortality in later life. Genome Biol. 2015;16:25-25. 
9. McCartney DL, Stevenson AJ, Walker RM, Gibson J, Morris SW, Campbell A, et al. Investigating the relationship between DNA methylation age acceleration and risk factors for Alzheimer's disease. Alzheimers Dement. 2018;10:429-437.

10. Perna L, Zhang Y, Mons U, Holleczek B, Saum K-U, Brenner H. Epigenetic age acceleration predicts cancer, cardiovascular, and allcause mortality in a German case cohort. Clin Epigenetics. 2016;8:64.

11. Horvath S, Ritz BR. Increased epigenetic age and granulocyte counts in the blood of Parkinson's disease patients. Aging. 2015;7:1130-42.

12. Chen BH, Marioni RE, Colicino E, Peters MJ, Ward-Caviness CK, Tsai PC, et al. DNA methylation-based measures of biological age: meta-analysis predicting time to death. Aging. 2016;8:1844-1865.

13. Lu AT, Quach A, Wilson JG, Reiner AP, Aviv A, Raj K, et al. DNA methylation GrimAge strongly predicts lifespan and healthspan. Aging. 2019;11:303-327.

14. Tucker-Drob EM. Neurocognitive functions and everyday functions change together in old age. Neuropsychology. 2011;25:368-77.

15. Raz N, Rodrigue KM. Differential aging of the brain: patterns, cognitive correlates and modifiers. Neurosci Biobehav Rev. 2006;30:730-48.

16. Calvin CM, Deary IJ, Fenton C, Roberts BA, Der G, Leckenby N, et al. Intelligence in youth and all-cause-mortality: systematic review with meta-analysis. Int J Epidemiol. 2011;40:626-44.

17. Calvin CM, Batty GD, Der G, Brett CE, Taylor A, Pattie A, et al. Childhood intelligence in relation to major causes of death in 68 year follow-up: prospective population study. Brit Med J. 2017; 357:j2708.

18. Čukić I, Brett CE, Calvin CM, Batty GD, Deary IJ. Childhood IQ and survival to 79: follow-up of $94 \%$ of the Scottish Mental Survey 1947. Intelligence. 2017;63:45-50.

19. Wraw C, Deary IJ, Gale CR, Der G. Intelligence in youth and health at age 50. Intelligence. 2015;53:23-32.

20. Gale CR, Booth T, Starr JM, Deary IJ. Intelligence and socioeconomic position in childhood in relation to frailty and cumulative allostatic load in later life: the Lothian Birth Cohort 1936. J Epidemiol Community Health. 2016;70:576-82.

21. Wraw C, Der G, Gale CR, Deary IJ. Intelligence in youth and health behaviours in middle age. Intelligence. 2018;69:71-86.

22. Karama S, Bastin ME, Murray C, Royle NA, Penke L, Maniega SMunoz, et al. Childhood cognitive ability accounts for associations between cognitive ability and brain cortical thickness in old age. Mol Psychiatry. 2014;19:555-9.

23. Deary IJ, Bastin ME, Pattie A, Clayden JD, Whalley LJ, Starr JM, et al. White matter integrity and cognition in childhood and old age. Neurology. 2006;66:505-12.

24. Valdés Hernández MDC, Booth T, Murray C, Gow AJ, Penke L, Morris Z, et al. Brain white matter damage in aging and cognitive ability in youth and older age. Neurobiol aging. 2013;34:2740-2747.

25. Deary IJ, Leaper SA, Murray AD, Staff RT, Whalley LJ. Cerebral white matter abnormalities and lifetime cognitive change: a 67year follow-up of the Scottish Mental Survey of 1932. Psychol Aging. 2003;18:140-8.

26. McHutchison CA, Backhouse EV, Cvoro V, Shenkin SD, Wardlaw JM. Education, socioeconomic status, and intelligence in childhood and stroke risk in later life: a meta-analysis. Epidemiology. 2017;28:608-618.

27. Backhouse EV, McHutchison CA, Cvoro V, Shenkin SD, Wardlaw JM. Early life risk factors for cerebrovascular disease: a systematic review and meta-analysis. Neurology. 2017;88:976-984.

28. Stevenson AJ, McCartney DL, Hillary RF, Redmond P, Taylor $\mathrm{AM}$, Zhang Q, et al., Childhood intelligence attenuates the association between biological ageing and health outcomes in later life. https://www.biorxiv.org/content/10.1101/588293v1. 2019.

29. Deary IJ, Gow AJ, Taylor MD, Corley J, Brett C, Wilson V, et al. The Lothian Birth Cohort 1936: a study to examine influences on cognitive ageing from age 11 to age 70 and beyond. BMC Geriatr. 2007;7:28-28.

30. Taylor AM, Pattie A, Deary IJ. Cohort profile update: the Lothian Birth Cohorts of 1921 and 1936. Int J Epidemiol. 2018;47:1042-1042r.

31. Shah S, McRae AF, Marioni RE, Harris SE, Gibson J, Henders AK, et al. Genetic and environmental exposures constrain epigenetic drift over the human life course. Genome Res. 2014;24:1725-33.

32. Zhang Q, Marioni RE, Robinson MR, Higham J, Sproul D, Wray $\mathrm{NR}$, et al. Genotype effects contribute to variation in longitudinal methylome patterns in older people. Genome Med. 2018;10:75.

33. Horvath S. DNA methylation age of human tissues and cell types. Genome Biol. 2013;14:R115.

34. Velissaris D, Pantzaris N, Koniari I, Koutsogiannis N, Karamouzos V, Kotroni I, et al. C-Reactive protein and frailty in the elderly: a literature review. J Clin Med Res. 2017;9:461-465.

35. Takata Y, Ansai T, Soh I, Awano S, Sonoki K, Akifusa S, et al. Serum albumin levels as an independent predictor of 4-year mortality in a community-dwelling 80 -year-old population. Aging Clin Exp Res. 2010;22:31-5.

36. Odden MC, Shlipak MG, Tager IB. Serum creatinine and functional limitation in elderly persons. J Gerontol Ser A, Biol Sci Med Sci. 2009;64:370-376.

37. Cabrera MA, de Andrade SM, Dip RM. Lipids and all-cause mortality among older adults: a 12-year follow-up study. Scientific World J. 2012;2012:930139.

38. Kadoglou NPE, Biddulph JP, Rafnsson SB, Trivella M, Nihoyannopoulos P, Demakakos P. The association of ferritin with cardiovascular and all-cause mortality in community-dwellers: the English longitudinal study of ageing. PLoS ONE. 2017; 12: 0178994.

39. Weiss A, Beloosesky Y, Boaz M, Yalov A, Kornowski R, Grossman E. Body mass index is inversely related to mortality in elderly subjects. J Gen Intern Med. 2008;23:19-24.

40. Celis-Morales CA, Welsh P, Lyall DM, Steell L, Petermann F, Anderson J, et al. Associations of grip strength with cardiovascular, respiratory, and cancer outcomes and all cause mortality: prospective cohort study of half a million UK Biobank participants. Br Med J. 2018;361:k1651.

41. Sin DD, Wu L, Man SF. The relationship between reduced lung function and cardiovascular mortality: a population-based study and a systematic review of the literature. Chest. 2005;127:1952-9.

42. Mannino DM, Davis KJ. Lung function decline and outcomes in an elderly population. Thorax. 2006;61:472-477.

43. Bates, D, Mächler M, Bolker B, Walker S, Fitting linear mixedeffects models using lme4. J Stat Softw. 2015;1:1-48.

44. Wardlaw JM, Bastin ME, Valdes Hernandez MC, Maniega SM, Royle NA, Morris Z, et al. Brain aging, cognition in youth and old age and vascular disease in the Lothian Birth Cohort 1936: rationale, design and methodology of the imaging protocol. Int $\mathrm{J}$ Stroke. 2011;6:547-59.

45. Valdes Hernandez Mdel C, Gallacher PJ, Bastin ME, Royle NA, Maniega SM, Deary IJ, et al. Automatic segmentation of brain white matter and white matter lesions in normal aging: comparison of five multispectral techniques. Magn Reson Imaging. 2012;30:222-9.

46. Penke L, Maniega SM, Murray C, Gow AJ, Valdés Hernández MC, Clayden JD, et al. A General factor of brain white matter integrity predicts information processing speed in healthy older people. J Neurosci. 2010;30:7569-7574.

47. Deary IJ, Gow AJ, Taylor MD, Corley J, Brett C, Wilson V, et al. The Lothian Birth Cohort 1936: a study to examine influences on cognitive ageing from age 11 to age 70 and beyond. BMC Geriatr. 2007;7:28. 
48. Benjamini Y, Hochberg Y. Controlling the false discovery rate: a practical and powerful approach to multiple testing. J R Stat Soc Ser B. 1995;57:289-300.

49. Franceschi C, Bonafe M, Valensin S, Olivieri F, De Luca M, Ottaviani E, et al. Inflamm-aging. An evolutionary perspective on immunosenescence. Ann N. Y Acad Sci. 2000;908:244-54.

50. Weverling-Rijnsburger AW, Blauw GJ, Lagaay AM, Knook DL, Meinders AE, Westendorp RG. Total cholesterol and risk of mortality in the oldest old. Lancet. 1997;350:1119-23.

51. Paul RF, Hassan M, Nazar HS, Gillani S, Afzal N, Qayyum I. Effect of body mass index on serum leptin levels. J Ayub Med Coll Abbottabad. 2011;23:40-3.

52. Al Maskari MY, Alnaqdy AA. Correlation between Serum Leptin Levels, Body Mass Index and Obesity in Omanis. Sultan Qaboos Univ Med J. 2006;6:27-31.

53. Horn JL, Cattell RB. Age differences in fluid and crystallized intelligence. Acta Psychol. 1967;26:107-29.

54. Dykiert D, Deary IJ. Retrospective validation of WTAR and NART scores as estimators of prior cognitive ability using the Lothian Birth Cohort 1936. Psychol Assess. 2013;25:1361-6.

55. Zhao, L, Matloff W, Ning K, Kim H, Dinov ID, Toga AW, Agerelated differences in brain morphology and the modifiers in middle-aged and older adults. Cereb Cortex. 2018;29:4169-93.

56. Aycheh HM, Seong JK, Shin JH, Na DL, Kang B, Seo SW, et al. Biological brain age prediction using cortical thickness data: a large scale cohort study. Front Aging Neurosci. 2018;10:252.

57. Dickie DA, Karama S, Ritchie SJ, Cox SR, Sakka E, Royle NA, et al. Progression of white matter disease and cortical thinning are not related in older community-dwelling subjects. Stroke. 2016;47:410-416.

58. Sachdev PS, Wen W, Christensen H, Jorm AF. White matter hyperintensities are related to physical disability and poor motor function. J Neurol Neurosurg Psychiatry. 2005;76:362-367.
59. Salarirad S, Staff RT, Fox HC, Deary IJ, Whalley L, Murray AD. Childhood intelligence and brain white matter hyperintensities predict fluid intelligence age 78-81 years: a 1921 Aberdeen birth cohort study. Age Ageing. 2011;40:562-7.

60. Wardlaw JM, Chappell FM, Valdés Hernández MDC, Makin SDJ, Staals J, Shuler K, et al. White matter hyperintensity reduction and outcomes after minor stroke. Neurology. 2017;89:1003-1010.

61. Debette S, Markus HS. The clinical importance of white matter hyperintensities on brain magnetic resonance imaging: systematic review and meta-analysis. Br Med J. 2010;341:c3666.

62. Onyike CU. Psychiatric aspects of dementia. Continuum. 2016; 22:600-614.

63. Ohi K, Sumiyoshi C, Fujino H, Yasuda Y, Yamamori H, Fujimoto $\mathrm{M}$, et al. Genetic overlap between general cognitive function and schizophrenia: a review of cognitive GWASs. Int J Mol Sci. 2018;19:3822.

64. Meijsen JJ, Campbell A, Hayward C, Porteous DJ, Deary IJ, Marioni RE, et al. Phenotypic and genetic analysis of cognitive performance in Major Depressive Disorder in the Generation Scotland: Scottish Family Health Study. Transl Psychiatry. 2018;8:63.

65. Sabia S, Elbaz A, Dugravot A, Head J, Shipley M, HaggerJohnson G, et al. Impact of smoking on cognitive decline in early old age: the Whitehall II cohort study. Arch Gen psychiatry. 2012;69:627-635.

66. Gellert C, Schottker B, Brenner H. Smoking and all-cause mortality in older people: systematic review and meta-analysis. Arch Intern Med. 2012;172:837-44.

67. Power MC, Deal JA, Sharrett AR, Jack Jr CR, Knopman D, Mosley TH, et al. Smoking and white matter hyperintensity progression: the ARIC-MRI study. Neurology. 2015;84:841-848.

68. Zhang Y, Elgizouli M, Schöttker B, et al. DNA methylation markers predict lung cancer incidence. Clin Epigenetics. 2016; $8: 127-127$

\section{Affiliations}

\section{Robert F. Hillary ${ }^{1}$ - Anna J. Stevenson $\mathbb{1}^{1} \cdot$ Simon R. Cox $\mathbb{D}^{2,3} \cdot$ Daniel L. McCartney ${ }^{1} \cdot$ Sarah E. Harris $\mathbb{1}^{2,3} \cdot$ Anne Seeboth $^{1}$ - Jon Higham ${ }^{4}$. Duncan Sproul ${ }^{4,5} \cdot$ Adele M. Taylor $^{2,3} \cdot$ Paul Redmond $^{2,3} \cdot$ Janie Corley $^{2,3}$. Alison Pattie ${ }^{2,3} \cdot$ Maria del. C. Valdés Hernández ${ }^{2,6} \cdot$ Susana Muñoz-Maniega ${ }^{2,6} \cdot$ Mark E. Bastin $^{2,6}$. Joanna M. Wardlaw $\mathbb{D}^{2,6,7}$. Steve Horvath $\mathbb{D}^{8,9} \cdot$ Craig W. Ritchie ${ }^{10}$. Tara L. Spires-Jones ${ }^{7,11}$. Andrew M. McIntosh $\mathbb{1}^{2,12} \cdot$ Kathryn L. Evans ${ }^{1,2} \cdot \operatorname{lan}$ J. Deary ${ }^{2,3} \cdot$ Riccardo E. Marioni ${ }^{1,2}$}

1 Centre for Genomic and Experimental Medicine, Institute of Genetics and Molecular Medicine, University of Edinburgh, Edinburgh, UK

2 Centre for Cognitive Ageing and Cognitive Epidemiology, University of Edinburgh, Edinburgh, UK

3 Department of Psychology, University of Edinburgh, Edinburgh, UK

4 Medical Research Council Human Genetics Unit, Medical Research Council Institute of Genetics and Molecular Medicine, University of Edinburgh, Edinburgh, UK

5 Edinburgh Cancer Research Centre, Medical Research Council Institute of Genetics and Molecular Medicine, University of Edinburgh, Edinburgh, UK

6 Department of Neuroimaging Sciences, Centre for Clinical Brain Sciences, University of Edinburgh, Edinburgh, UK
7 UK Dementia Research Institute, Edinburgh Medical School, University of Edinburgh, Edinburgh, UK

8 Department of Human Genetics, David Geffen School of Medicine, University of California Los Angeles, Los Angeles, California, USA

9 Department of Biostatistics, Fielding School of Public Health, University of California Los Angeles, Los Angeles, California, USA

10 Edinburgh Dementia Prevention, Centre for Clinical Brain Sciences, University of Edinburgh, Edinburgh, UK

11 Centre for Discovery Brain Sciences, University of Edinburgh, Edinburgh, UK

12 Division of Psychiatry, Centre for Clinical Brain Sciences, University of Edinburgh, Edinburgh, UK 